\title{
DOR NEUROPÁTICA EM PACIENTES COM HIVIAIDS EM USO DE TERAPIA ANTIRRETROVIRAL
}

\author{
NEUROPATHIC PAIN IN HIVIAIDS PATIENTS USING \\ ANTIRETROVIRAL THERAPY
}

Andressa de Souza1 ${ }^{1}$, Rafael Braz da Silva², Jairo Alberto Dussán-Sarria ${ }^{3}$, Alexandre Ramos Lazzarotto ${ }^{1}$

\section{RESUMO}

Clin Biomed Res. 2016;36(3):156-164

1 Programa de Pós-graduação em Saúde e Desenvolvimento Humano, Centro Universitário La Salle. Canoas, RS, Brasil.

2 Curso de Psicologia e Processos de Prevenção e Promoção da Saúde, Centro Universitário La Salle. Canoas, RS, Brasil.

3 Residência Médica em Anestesiologia, Hospital de Clínicas de Porto Alegre. Porto Alegre, RS, Brasil.

Autor correspondente:

Andressa de Souza

andressasz@gmail.com

Centro Universitário La Salle

Avenida Victor Barreto, 2288.

92010-000, Canoas, RS, Brasil.
A infecção pelo vírus da imunodeficiência humana (HIV) representa um dos maiores problemas de saúde da atualidade em virtude de seu caráter pandêmico e gravidade. O uso da terapia antirretroviral está associado ao desenvolvimento de diferentes tipos de dor, sendo a dor neuropática uma delas. A dor neuropática ocorre pela lesão do sistema nociceptivo, que envolve mudanças moleculares, fisiológicas e anatômicas. Considerando que a infecção por HIV gera custos elevados para o sistema de saúde, e que suas comorbidades elevam ainda mais esses custos, a compreensão dos mecanismos da dor nessa população possibilita uma melhor assistência e uma redução da carga para o sistema. A dor neuropática pode apresentar diferentes possíveis mecanismos fisiopatológicos envolvidos, tornando-se um desafio para o tratamento de pacientes com HIV. A compreensão sobre a neurofisiologia da dor neuropática e o HIV pode promover melhores abordagens aos pacientes e a redução de comorbidades associadas, com impacto na qualidade de vida.

Palavras-chave: Dor neuropática; HIV; terapia antirretroviral

\begin{abstract}
Human immunodeficiency virus (HIV) infection is a major health problem nowadays due to its pandemic character and severity. The use of antiretroviral therapy is associated with the development of different types of pain, and neuropathic pain is one of them. Neuropathic pain is caused by an injury to the nociceptive system, which involves molecular, physiological and anatomical changes. Considering that HIV infection generates high costs for the health system, and that its comorbidities raise such costs even more, understanding the mechanisms of pain in this population can result in better care practices and reduction of burden to the system. Neuropathic pain may present different pathophysiological mechanisms becoming a challenge for HIV treatment. The understanding of the neurophysiology of neuropathic pain and the HIV can promote better patient approaches and the reduction of associated comorbidities with an impact on quality of life.
\end{abstract}

Keywords: Neuropathic pain; HIV; antiretroviral therapy

O vírus da imunodeficiência humana (HIV) pertence à família Retroviridae, subfamília Lentiviridae. Ele causa efeitos citopáticos em curto prazo e uma infecção longitudinal persistente que culmina em um quadro clínico de imunodeficiência, que corresponde à síndrome da imunodeficiência adquirida (AIDS). A sua principal característica é a supressão profunda da imunidade mediada por células T, que torna o indivíduo suscetível a infecções oportunistas, neoplasias secundárias e doenças neurológicas que, se não forem combatidas, levam-no inevitavelmente ao óbito¹.

A Organização Mundial da Saúde (OMS) informou que, ao final de 2013, havia $35(33,1-37,2)$ milhões de pessoas vivendo com a infecção no mundo². Embora a incidência global tenha sido estabilizada em 2,1 milhões em 2013, o número de pacientes que recebem a terapia antirretroviral combinada (TARV) 
continua aumentando - estima-se que 12,9 milhões de pacientes receberam o tratamento até dezembro de 2013. Conforme o Boletim Epidemiológico HIVAIDS $^{3}$ do Ministério da Saúde, havia, em 2014, 734 mil pessoas vivendo com HIVIAIDS no Brasil. A taxa de detecção no Brasil, em média, é de 20,5 casos para cada 100 mil habitantes. Na região Sul, a taxa é de 31,1 casos para cada 100 mil habitantes, e, no Rio Grande do Sul, de 41,3 casos para cada 100 mil habitantes. Porto Alegre, capital do estado do Rio Grande do Sul, teve a maior taxa de detecção (96,2 por 100 mil habitantes) de casos de AIDS entre as capitais brasileiras ${ }^{2}$.

A partir do uso da TARV, mediada pela introdução de inibidores da protease em 1997 no Brasil, houve uma mudança positiva na expectativa de vida dos portadores do HIVIAIDS ${ }^{4}$. Independentemente dos diferentes fenótipos associados à progressão da infecção pelo HIV, a TARV é necessária para suprimir a replicação viral. Na ausência dela ou na má adesão do paciente, ocorre o aumento da replicação e, como consequência, a depleção das células T CD4+, imunodeficiência progressiva e AIDS 5 .

Infelizmente, o uso continuado da TARV causa efeitos adversos de tipo metabólico (por exemplo, diabetes tipo II e hipercolesterolemia) ${ }^{6}$, antropométrico (por exemplo, lipodistrofia) ${ }^{6}$ e neurológico (por exemplo, neuropatia periférica) ${ }^{7}$. Nesse contexto, a neuropatia ainda se destaca pela carência de conhecimentos sobre a dor associada ao tipo de fármaco ou esquema terapêutico utilizado pelo paciente.
Merlin et al. ${ }^{8}$ mostraram que $61,9 \%$ dos pacientes com HIV apresentaram queixas de dor, e que a dor estava correlacionada a abuso de substâncias ou doenças psiquiátricas. Além disso, foi descrito que quanto mais grave é o estágio da doença e pior o estado de saúde, maior é a intensidade da dor ${ }^{8}$. Diante desse contexto, o objetivo deste estudo foi realizar uma revisão da literatura científica sobre o desenvolvimento da dor neuropática em pacientes com HIVIAIDS que fazem uso contínuo da TARV.

\section{MÉTODO}

O estudo envolveu uma revisão sistemática na qual os artigos foram localizados de acordo com o ano de publicação (2000 a 2015) nos seguintes idiomas: português, inglês e espanhol. Os artigos foram selecionados nas bases de dados SciELO e PubMed. Os unitermos de busca foram: dor neuropática, HIV e terapia antirretroviral, além dos termos em inglês neuropathic pain, HIV e antiretroviral therapy. Os estudos foram classificados de acordo com os níveis de evidência do Centre for Evidence-Based Medicine de Oxford $^{9}$. A Figura 1 ilustra a seleção de artigos. Além disso, outros estudos considerados importantes na área da dor foram utilizados para melhor embasar a revisão. Alguns artigos encontrados na PubMed também estavam no EMBASE. Após selecionar os artigos, enfocaram-se os seguintes tópicos: dor neuropática e sua fisiopatogenia, fisiopatologia da dor neuropática em pacientes com HIV/Aids em uso de TARV e clínica do paciente. Abordamos então os desafios para o tratamento desses pacientes.

Palavras-chave: Dor Neuropática, HIV, Terapia Antirretroviral.

Keywords: Neuropathic Pain, HIV, Antiretroviral Therapy.

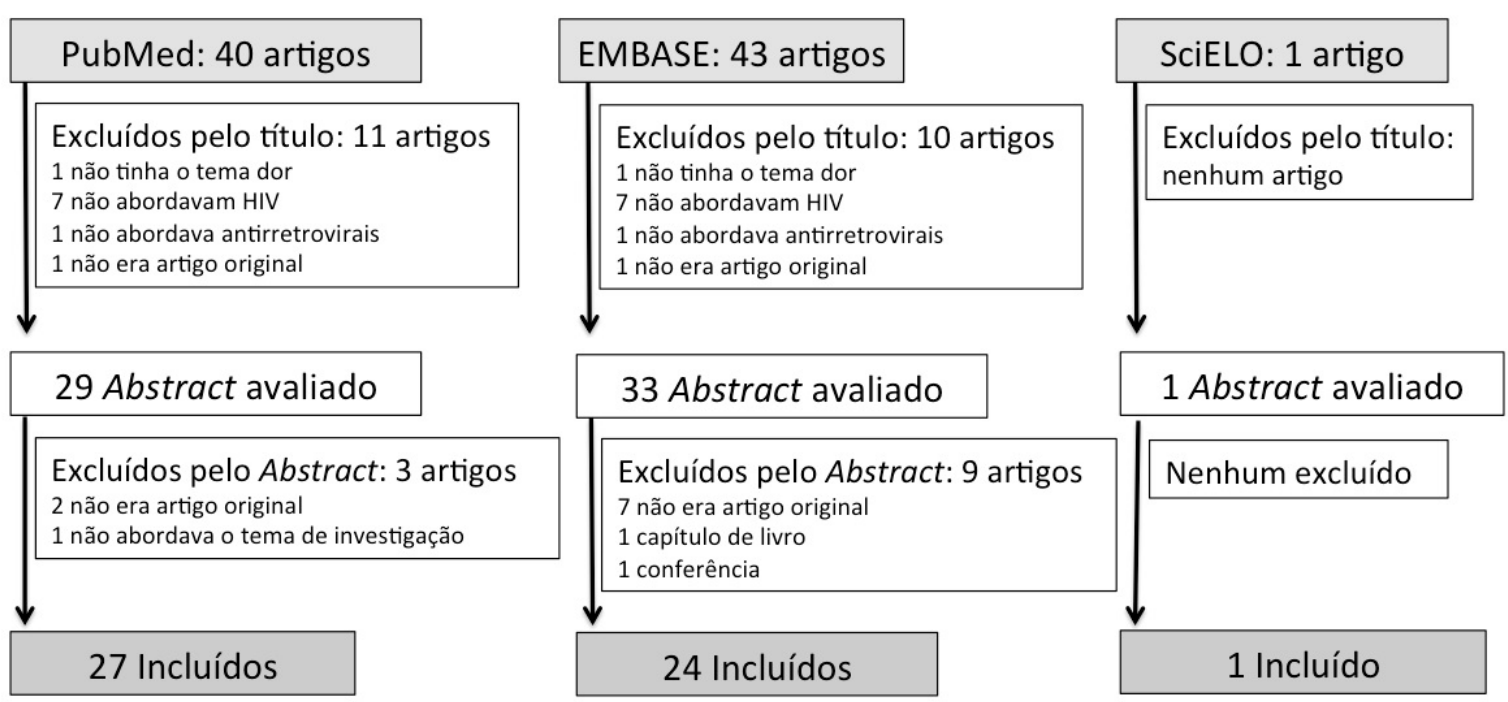

Figura 1: Estratégia de busca e seleção de artigos. 


\section{DOR NEUROPÁTICA}

A dor é um sintoma que, agudamente, tem uma função adaptativa para os organismos, mas, quando ultrapassa a duração de três meses, é considerada crônica. Pode assim deformar a fisiologia e o comportamento, tornando-se desadaptativa e patológica. A dor crônica per se é uma doença debilitante, de difícil manejo, que traz prejuízos sociais e econômicos. Quando a sua origem é a lesão direta do nervo ou do sistema somatossensorial, é conhecida como dor neuropática ${ }^{10}$, que, muitas vezes, torna-se resistente aos tratamentos atualmente disponíveis ${ }^{11}$. Essa dor é relativamente comum, ocorrendo em aproximadamente 6 a $8 \%$ da população em geral ${ }^{12,13}$.

As estimativas de prevalência da dor em indivíduos infectados pelo HIV variam de 30 a $90 \%$, com prevalência crescente em fases mais avançadas da doença ${ }^{14}$. Em 1997, Larue et al. ${ }^{15}$ constataram que $30 \%$ dos pacientes ambulatoriais e $62 \%$ dos pacientes internados com HIV relataram dor relacionada ao HIV, e que a gravidade dessa dor foi significativamente subestimada pelos médicos que prestam cuidados a pacientes soropositivos. Em adultos com HIV, a dor neuropática é mais frequente em homens com baixos níveis de CD4 ou carga viral aumentada. Nessa população, $80 \%$ dos pacientes referem ter experimentado dor severa, que interfere no humor, na qualidade de vida e nas habilidades de trabalho ${ }^{16}$.

Esses resultados corroboram um estudo sobre HIV realizado na Índia, que mostrou que $66,7 \%$ dos pacientes internados e $24,5 \%$ dos pacientes ambulatoriais queixaram-se de dor ${ }^{17}$. Essa discrepância pode estar relacionada ao fato de que muitos pacientes estavam na fase 3 ou 4 de HIV (90\% versus $40 \%)$. Os locais primários de dor listados foram cabeça, pernas e $\operatorname{costas}^{18}$. Em outro estudo sobre dor em pacientes indigentes com HIV, 91,2\% relataram dor, com $53,7 \%$ classificando a sua dor como intensa ${ }^{17}$. Os locais mais frequentes de dor nessa população eram pernas e parte inferior das $\operatorname{costas}^{17}$.

Entre as causas de dor em pacientes com HIV, existem as não relacionadas ao HIV, como dor lombar, com prevalência de $24 \%$; as relacionadas ao HIV, como neuropatia por HIV, infecções oportunistas e sarcoma de Kaposi, com prevalência de $30 \%$; as secundárias ao tratamento do HIV, como neuropatia devido à TARV ou quimioterapia, com prevalência de $4 \%$; e as de causa idiopática, com prevalência de $37 \%{ }^{19}$. Apesar da melhora na efetividade e tolerabilidade da TARV, a neuropatia sensitiva dolorosa no HIV ainda é comum e resulta em afastamentos do trabalho e limitações das atividades da vida cotidiana ${ }^{20}$.

Na população pediátrica, um estudo observacional realizado na África do Sul avaliou a dor em crianças com HIV entre 3 e 7 anos de idade em uso de TARV.
Observou-se que metade delas já apresentavam dor, existindo uma associação inversa entre número de CD4 e dor. Porém, destacou-se que o tipo de dor mais frequente foi a nociceptiva. A dor em crianças ainda não é adequadamente tratada, sendo paracetamol o principal analgésico utilizado nessa população ${ }^{21}$.

\section{FISIOPATOLOGIA DA DOR NEUROPÁTICA EM PACIENTES COM HIVIAIDS EM USO DE TARV}

Estudo realizado em ambulatório para atendimento de pacientes com HIV reportou que $83,7 \%$ dos pacientes com HIV apresentam algum tipo de dor, sendo que $38,76 \%$ dos pacientes avaliados foram diagnosticados com dor crônica. Embora $82 \%$ dos pacientes utilizassem medicamentos para essa condição dolorosa, somente $23,58 \%$ obtinham alívio da dor. No entanto, nesse estudo, não foi descrito o uso da TARV22.

O significativo progresso da pesquisa básica e clínica tem sido essencial na caracterização dos mecanismos da dor neuropática nesses pacientes. Acredita-se que essa condição pode ser resultante de uma lesão axonal advinda da ligação do envelope glicoproteico viral aos receptores de quimiocinas e desregulação de macrófagos, os quais liberam citocinas pró-inflamatórias neurotóxicas ${ }^{23,24}$. A neuropatia associada ao uso dos medicamentos talvez possa ser mediada pela inibição da síntese de DNA, que interfere na função mitocondria ${ }^{25}$. Com a toxicidade medicamentosa, o início dos sintomas tende a ser súbito e associado ao início da TARV, e, geralmente, o pico dos sintomas acontece nos 3 primeiros meses ${ }^{26,27}$.

Estudos relatam o envolvimento da proteína gp120, descrita como a glicoproteína viral que recobre o envelope e que, uma vez em contato com a superfície da célula, pode produzir danos ${ }^{28}$. Agp120 exerce efeitos neurotóxicos diretos e indiretos no sistema nervoso através de fatores pró-inflamatórios ${ }^{29}$. A participação direta da gp120 na gênese da dor neuropática tem sido sugerida. A administração intratecal de recombinante da gp120 induz hiperalgesia térmica e mecânica, além de intensa alodinia ${ }^{30}$. Outros estudos também descrevem um envolvimento da micróglia, em que produtos liberados pela micróglia infectada, denominadas virotoxinas, ativariam astrócitos, liberação de quimiocinas e, consequentemente, neurotoxicidade ${ }^{31}$.

Esses danos neuronais podem promover uma sensibilização central, processo por meio do qual um estado de hiperexcitabilidade é estabelecido no sistema nervoso central, conduzindo a um aumento das respostas nociceptivas (dolorosas) ${ }^{32}$. Embora muitos mecanismos têm sido propostos para a sensibilização central, existem pelo menos três aspectos principais envolvidos na sensibilização: 
transmissão glutamatérgica/N-metil-D-aspartato (NMDA), perda do controle inibitório (desinibição) e interações glia-neurônio ${ }^{33}$. Em situações normais, há um equilíbrio do sistema. Entretanto, na configuração da lesão, o aumento da liberação de neurotransmissores a partir de nociceptores aferentes primários suficientemente despolariza neurônios pós-sinápticos para ativar os receptores de NMDA em neurônios de segunda ordem ${ }^{34}$. O consequente aumento do influxo de cálcio pode fortalecer conexões sinápticas entre os nociceptores e o corno dorsal, neurônios de transmissão da dor que, por sua vez, agravam respostas a estímulos nocivos ${ }^{33}$. Uma série de vias de sinalização e sistemas de segundo mensageiro (como proteína quinase $A$, proteína quinase $\mathrm{C}$, fosfatidilinositol 3-fosfato) está envolvida na excitabilidade desses neurônios ${ }^{35,36}$. O principal tipo de transmissão sináptica inibitória no corno dorsal é mediado pelo ácido gama-aminobutírico (GABA) e receptores de glicina. Sob condições normais, o controle inibitório sobre o sistema de dor é muito poderoso, servindo como um "controle do portão" para manter o equilíbrio entre vias excitatórias e inibitórias ${ }^{37,38}$. Porém, na definição da lesão, o processo de inibição pode ser perdido, resultando na perturbação do equilíbrio e levando a uma anormal sensibilidade à dor ${ }^{39,40}$.

Além disso, as células gliais estão envolvidas na formação de sinapses, na plasticidade e na rápida condução de potenciais de ação, bem como nas funções imunológicas do sistema nervoso ${ }^{41}$. A lesão/inflamação do nervo periférico promove liberação de neurotransmissores e neuropeptídios que estimulam células gliais. A ativação da micróglia ocorre em poucos minutos, mas pode ser de longa duração ${ }^{42}$. A liberação de fatores neurotróficos derivados do cérebro (BDNF), e uma série de citocinas, como TNF, IL-1 e IL-6, que, através de seus receptores, expressam por neurônios no corno dorsal da medula, promovem um aumento da excitabilidade e dor reforçada em resposta tanto ao estímulo nocivo (hiperalgesia) quanto ao estímulo inócuo (alodinia) ${ }^{32,36,43,44}$.

Já foi descrito que existe um envolvimento do próprio vírus ou da TARV nos processos de transdução citoplasmática envolvidos na inflamação ${ }^{45}$. 0 início do uso da TARV promove aumento dos níveis de interleucinas ${ }^{46}$, sendo que foi observado um envolvimento do TNF-alfa na dor neuropática induzida por inibidor de transcriptase reversa ${ }^{47}$. Além disso, o bloqueio da sinalização de moléculas pró-inflamatórias foi capaz de reduzir a dor neuropática envolvida com alodinia mecânica ${ }^{48}$, reforçando o papel importante dos processos inflamatórios na resposta dolorosa.

Essa sensibilidade exacerbada foi referida principalmente no uso da TARV, que afeta neurônios de pequeno diâmetro do gânglio da raiz dorsal da medula ${ }^{49}$. Além disso, a severidade da neuropatia foi correlacionada a um menor volume de massa cinzenta cortical ${ }^{50}$.

Variações genéticas também parecem estar envolvidas na susceptibilidade da neuropatia distal ${ }^{51}$. O polimorfismo no gene da Catecol O-Metiltransferase (COMT) altera o fenótipo da neuropatia sensitiva no HIV, aumentando a chance de dor neuropática distal. Há uma implicação por esse genótipo também estar associado ao metabolismo de alguns fármacos, incluindo opioides ${ }^{52}$. Preditores de desenvolvimento da dor em pacientes com HIV são a presença de alterações no exame neurológico compatíveis com a presença de neuropatia e história de abuso ou dependência de opioides ${ }^{53}$.

\section{CLÍNICA DO PACIENTE}

Os pacientes com dor neuropática podem apresentar sintomas positivos e negativos. Entre os positivos, destacam-se parestesia, disestesia e hiperestesia (algesia e alodinia). Entre os sintomas negativos, destacam-se déficits sensoriais (hipoestesia e hipoalgesia), fraqueza e mudanças nos reflexos ${ }^{54}$. Essa dor pode ser classificada com base na localização da lesão como de origem central ou periférica. As lesões periféricas englobam mudanças na excitabilidade do nervo periférico e gânglio da raiz dorsal, enquanto lesões centrais estão relacionadas a mudanças em neurônios da medula espinal, sistemas descendentes inibitórios e mudanças plásticas corticais ${ }^{55}$.

Estudos descrevem os mecanismos neurofisiológicos envolvidos nessa dor como impulsos excessivos (inputs) provenientes da periferia com mudanças plásticas em neurônios de segunda ordem e aumento dos campos receptivos ${ }^{10}$. Isso resulta em um aumento dos impulsos nociceptivos ascendentes, ativação das vias descendentes facilitatórias no bulbo rostroventral e substância cinzenta periaqueduta $\left.\right|^{56}$, e redução das vias descendentes inibitórias ${ }^{57}$. Todos esses mecanismos contribuem para a dificuldade do manejo terapêutico e farmacológico no tratamento dessa condição.

Mais especificamente, os processos periféricos estão envolvidos com atividade ectópica do nervo lesionado, expressão periférica anormal dos canais de cálcio, supersensibilidade noradrenérgica e mudanças fenotípicas em neurônios do gânglio da raiz dorsal, em que se destacam alterações na expressão de neurotransmissores, neuromoduladores, receptores e canais iônicos ${ }^{54}$. Além disso, há interação neuroimune no local onde a lesão direta do nervo periférico promove resposta inflamatória local, ou "sopa" inflamatória, com liberação de mediadores 
como histamina, bradicinina, ATP, citocinas, entre outros, que contribuem para a alteração da resposta periférica ${ }^{58}$.

Além do HIV, a neuropatia pode se desenvolver como resultado de outras doenças, como diabetes, lesão física, neoplasias, uso de medicamentos, alcoolismo, doenças genéticas, doenças autoimunes ou deficiência de vitaminas ${ }^{59}$. Os sintomas clínicos da neuropatia periférica em HIV incluem parestesia bilateral e simétrica, ardência/queimação ou perda de sensibilidade. Os sintomas tendem a começar nos dedos dos pés, subir até os tornozelos e, eventualmente, chegar à parte inferior das pernas, em um padrão conhecido como distribuição de "meia". É comum haver perda ou diminuição de reflexos do tornozelo, dor e prejuízo na percepção de temperatura ${ }^{60}$. Os pacientes podem experimentar perda de equilíbrio, levando a quedas, ou dificuldades para caminhar, tomar banho ou se vestir61. Esses sintomas podem causar extremo desconforto, afetando o emprego, a saúde emocional, a independência e a qualidade de vida ${ }^{62}$.

A maioria dos pacientes com HIV sentem dor e usam vários métodos para aliviá-la, mas com analgesia inadequada ${ }^{63}$. A dor neuropática é fortemente influenciada por desordens psiquiátricas ${ }^{64}$. A detecção do tratamento para depressão nos pacientes com dor neuropática distal moderada e grave é importante para o tratamento bem-sucedido dessa alteração ${ }^{65}$. É frequente ocorrer complicações neurológicas e, por isso, os pacientes descontinuam a TARV, o que causa prejuízos ao seu quadro imunológico ${ }^{66}$.

\section{DESAFIOS NO TRATAMENTO DA DOR CRÔNICA NEUROPÁTICA EM PACIENTES COM HIVIAIDS EM USO DE TARV}

O tratamento dos pacientes com HIV e dor crônica neuropática apresenta desafios adicionais devido às características dessa população. Por um lado, a TARV está associada à dor neuropática pelos mecanismos acima descritos, devido a uma efetiva e eficaz diminuição da replicação viral. Ao mesmo tempo, a TARV apresenta múltiplas interações farmacológicas, de modo que as decisões clínicas de início de outros fármacos se tornam complexas. Pacientes com HIV têm prevalência alta de outras doenças médicas e psiquiátricas, e precisam de tratamento farmacológico que, potencialmente, poderia mudar a biodisponibilidade dos fármacos em uso. Ao mesmo tempo, as doenças psiquiátricas per se representam um fator de risco para a má aderência às terapias e para o aumento da intensidade da dor ${ }^{14}$.

Alguns estudos têm buscado alternativas farmacológicas para o tratamento da dor em pacientes com HIV, porém sem melhora com analgésicos convencionais. Uma delas é o uso de patch de capsaicina (alta dose), que possui menos interações farmacológicas por ser de via transdérmica e resultou em uma redução de até 3-4 pontos na escala análogo-visual da dor ${ }^{67}$. Também tem sido sugerido que a inibição da degradação de endocanabinoides poderia auxiliar no alívio da dor neuropática no HIV ${ }^{68}$. Outro destaque é o uso da TARV à base de estavudina (um antirretroviral e nucleosídeo sintético análogo à timidina) por 3 meses, que reduziu a gravidade da neuropatia distal, mas são necessários estudos em longo prazo $^{69}$, talvez por melhorar o quadro imunológico desses pacientes.

Existem terapias não farmacológicas apresentaram eficácia no tratamento da dor crônica, como: acupuntura $^{70}$, estimulação elétrica transcraniana ${ }^{71}$, estimulação magnética transcraniana ${ }^{72}$, atividade física ${ }^{73}$, exercícios fisioterapêuticos ${ }^{74}$, estimulação da medula espinhal ${ }^{75-77}$ e terapia cognitivo-comportamental ${ }^{78}$. Além disso, sabe-se da dificuldade de adesão a tratamentos pelo paciente com HIV, não somente à TARV, mas também ao tratamento para dor, seja farmacológico ou não farmacológico ${ }^{79}$. Esses dados demonstram a importância do entendimento dos vários fatores associados à dor no paciente com HIV para que se possa compreender melhor os processos neurobiológicos e aprofundar estudos que possibilitem novas terapêuticas para esses pacientes.

\section{CONCLUSÃO}

A dor crônica neuropática em pacientes com HIV é uma manifestação clínica de processo fisiopatológico progressivo e prevalente que leva a um prejuízo significativo na qualidade de vida dos pacientes. A caracterização clínica dessa população e a descrição das mudanças neurofisiológicas no processamento da dor em diferentes estágios da doença podem permitir a busca por novas abordagens profiláticas e terapêuticas não farmacológicas com alvos mais específicos. Portanto, é importante reconhecer que tanto os mecanismos de replicação viral quanto o uso da TARV em associação com processos inflamatórios contribuem para a lesão axonal responsável pela manifestação clínica da neuropatia dolorosa nessa população. Esses conhecimentos se tornam essenciais ao desafio de diminuir interações com a TARV e melhorar a qualidade de vida dos pacientes com HIV.

\section{Agradecimentos}

Centro Universitário La Salle Canoas. Edital CNPQUniversal (442479/2014-0).

\section{Fonte de financiamento}

Conselho Nacional de Desenvolvimento Científico e Tecnológico - CNPq (Universal 442479/2014-0). Centro Universitário La Salle. 


\section{REFERÊNCIAS}

1. Roitt IM, Delves PJ, Martin SJ, Burton DR. Fundamentos de Imunologia. Rio de Janeiro: Guanabara Koogan; 2013.

2. World Health Organization (WHO). Global Health Observatory (GHO) data. WHO; 2015. [citado em 12 Mar 2015]. Disponível em: http://www.who. int/gho/hiv/en/.

3. Brasil. Ministério da Saúde. Departamento de DST, Aids e Hepatites Virais. Boletim Epidemiológico HIVIAIDS 2014. Brasil: DST-AIDS; 2014. [citado em 19 Mar 2015]. Disponível em: http:// www.aids.gov.br/sites/default/files/ anexos/publicacao/2014/56677/ boletim_2014_final_pdf_15565.pdf

4. Johnson LF, Mossong J, Dorrington RE, Schomaker M, Hoffmann CJ, Keiser O, et al. Life expectancies of South African adults starting antiretroviral treatment: collaborative analysis of cohort studies. PLOS Med. 2013;10(4):e1001418. PMid:23585736. http://dx.doi. org/10.1371/journal.pmed.1001418.

5. Suvada J. Neuropathic and neurocongnitive complications of antiretroviral therapy among HIVinfected patients. Neuroendocrinol Lett. 2013;34(Suppl 1):5-11. PMid:24013599.

6. Grunfeld C. Understanding the complications of antiretroviral drugs. Clin Infect Dis. 2008;47(4):5756. PMid:18611156. http://dx.doi. org/10.1086/590155.

7. Nicholas PK, Corless IB, Evans LA. Peripheral neuropathy in HIV: an analysis of evidence-based approaches. J Assoc Nurses AIDS Care. 2014;25(4):318-29. PMid:24698331. http://dx.doi. org/10.1016/j.jana.2014.01.002.

8. Merlin JS, Cen L, Praestgaard A, Turner M, Obando A, Alpert C, et al. Pain and physical and psychological symptoms in ambulatory HIV patients in the current treatment era. J Pain Symptom Manage. 2012;43(3):638-45. PMid:22115794. http://dx.doi.org/10.1016/j. jpainsymman.2011.04.019.

9. Centre for Evidence Based Medicine (CEBM). Oxford. [citado em 12 Mar 2015]. Disponível em: www.cebm.net

10. Treede RD, Jensen TS, Campbell JN, Cruccu G, Dostrovsky JO,
Griffin JW, et al. Neuropathic pain: redefinition and a grading system for clinical and research purposes. Neurology. 2008;70(18):1630-5. PMid:18003941. http://dx.doi.org/10.1212/01. wnl.0000282763.29778.59.

11. Jensen TS, Gottrup H, Sindrup SH, Bach FW. The clinical picture of neuropathic pain. Eur J Pharmacol. 2001;429(1-3):1-11. PMid:11698022. http://dx.doi.org/10.1016/S00142999(01)01302-4.

12. Torrance N, Smith BH, Bennett MI, Lee AJ. The epidemiology of chronic pain of predominantly neuropathic origin. Results from a general population survey. J Pain. 2006;7(4):281-9. PMid:16618472. http://dx.doi.org/10.1016/j. jpain.2005.11.008.

13. Bouhassira $D$, Lantéri-Minet $M$, Attal N, Laurent B, Touboul C. Prevalence of chronic pain with neuropathic characteristics in the general population. Pain. 2008;136(3):3807. PMid:17888574. http://dx.doi. org/10.1016/j.pain.2007.08.013.

14. Krashin DL, Merrill JO, Trescot AM. Opioids in the management of HIV-related pain. Pain Physician. 2012;15(3 Suppl):ES157-68. PMid:22786454.

15. Larue F, Fontaine A, Colleau SM. Underestimation and undertreatment of pain in HIV disease: multicentre study. BMJ. 1997;314(7073):238. PMid:9001475. http://dx.doi. org/10.1136/bmj.314.7073.23.

16. Hitchcock SA, Meyer HP, Gwyther E. Neuropathic pain in AIDS patients prior to antiretroviral therapy. $S$ Afr Med J. 2008;98(11):889-92. PMid:19177897.

17. Miaskowski C, Penko JM, Guzman D, Mattson JE, Bangsberg DR, Kushel MB. Occurrence and characteristics of chronic pain in a community-based cohort of indigent adults living with HIV infection. J Pain. 2011;12(9):100416. PMid:21684218. http://dx.doi. org/10.1016/j.jpain.2011.04.002.

18. Nair SN, Mary TR, Prarthana S, Harrison P. Prevalence of pain in patients with HIVIAIDS: a crosssectional survey in a South Indian State. Indian J Palliat Care. 2009;15(1):67-70. PMid:20606859. http://dx.doi.org/10.4103/09731075.53550 .
19. Hewitt DJ, McDonald M, Portenoy RK, Rosenfeld B, Passik S, Breitbart W. Pain syndromes and etiologies in ambulatory AIDS patients. Pain. 1997;70(2-3):117-23. PMid:9150284. http://dx.doi.org/10.1016/S03043959(96)03281-2.

20. Ellis RJ, Rosario D, Clifford D, McArthur J, Simpson D, Alexander TJ, et al. Neuropathic pain contributes to an excess of unemployment and disability in HIV infection: the charter study. J Neurovirol. 2009;15:26-7.

21. Azam M, Campbell L, Ross A. Exploration of pain in children on antiretroviral treatment in a regional hospital in South Africa. S Afr Fam Pract. 2012;54(4):358-62. http://dx.doi. org/10.1080/20786204.2012.10874249.

22. Ebirim LN, Otokwala JG. Inadequate pain relief in ambulatory patients with human immunodeficiency virus disease in Port Harcourt. HIV AIDS (Auckl). 2013;5:199203. PMid:23976866. http://dx.doi. org/10.2147/HIV.S45150.

23. Verma S, Estanislao L, Simpson D. HIV-associated neuropathic pain: epidemiology, pathophysiology and management. CNS Drugs. 2005;19(4):325-34. PMid:15813646. http://dx.doi.org/10.2165/00023210200519040-00005.

24. Melli G, Keswani SC, Fischer A, Chen W, Höke A. Spatially distinct and functionally independent mechanisms of axonal degeneration in a model of HIV-associated sensory neuropathy. Brain. 2006;129(Pt 5):1330-8. PMid:16537566. http://dx.doi. org/10.1093/brain/awl058.

25. Pardo CA, McArthur JC, Griffin JW. HIV neuropathy: insights in the pathology of HIV peripheral nerve disease. J Peripher Nerv Syst. 2001;6(1):21-7. PMid:11293804. http://dx.doi.org/10.1046/j.15298027.2001.006001021.x.

26. McArthur JC, Brew BJ, Nath A. Neurological complications of HIV infection. Lancet Neurol. 2005;4(9):543-55. PMid:16109361. http://dx.doi.org/10.1016/S14744422(05)70165-4.

27. Dalakas MC. Peripheral neuropathy and antiretroviral drugs. J Peripher Nerv Syst. 2001;6(1):14-20. PMid:11293802. http://dx.doi.org/10.1046/j.15298027.2001.006001014.x. 
28. Kramer-Hämmerle $S$, Rothenaigner I, Wolff H, Bell JE, Brack-Werner R. Cells of the central nervous system as targets and reservoirs of the human immunodeficiency virus. Virus Res. 2005;111(2):194-213. PMid:15885841. http://dx.doi.org/10.1016/j. virusres.2005.04.009.

29. Kaul M, Garden GA, Lipton SA. Pathways to neuronal injury and apoptosis in HIV-associated dementia. Nature. 2001;410(6831):988-94. PMid:11309629. http://dx.doi. org/10.1038/35073667.

30. Milligan ED, Mehmert KK, Hinde JL, Harvey LO JR, Martin D, Tracey $\mathrm{KJ}$, et al. Thermal hyperalgesia and mechanical allodynia produced by intrathecal administration of the human immunodeficiency virus-1 (HIV-1) envelope glycoprotein, gp120. Brain Res. 2000;861(1):10516. PMid: 10751570 . http://dx.doi. org/10.1016/S0006-8993(00)02050-3.

31. Nath A, Hauser KF, Wojna V, Booze RM, Maragos W, Prendergast M, et al. Molecular basis for interactions of HIV and drugs of abuse. J Acquir Immune Defic Syndr. 2002;31(Suppl 2):S629. PMid:12394784. http://dx.doi. org/10.1097/00126334-20021001200006.

32. Woolf CJ. Evidence for a central component of post-injury pain hypersensitivity. Nature. 1983;306(5944):686-8. PMid:6656869. http://dx.doi.org/10.1038/306686a0.

33. Basbaum Al, Bautista DM, Scherrer G, Julius D. Cellular and molecular mechanisms of pain. Cell. 2009;139(2):267-84. PMid:19837031. http://dx.doi.org/10.1016/j. cell.2009.09.028.

34. Yaksh TL, Hua XY, Kalcheva I, Nozaki-Taguchi N, Marsala M. The spinal biology in humans and animals of pain states generated by persistent small afferent input. Proc Natl Acad Sci USA. 1999;96(14):76806. PMid:10393880. http://dx.doi. org/10.1073/pnas.96.14.7680.

35. Latremoliere A, Woolf CJ. Central sensitization: a generator of pain hypersensitivity by central neural plasticity. J Pain. 2009;10(9):895926. PMid:19712899. http://dx.doi. org/10.1016/j.jpain.2009.06.012.

36. Guo W, Wang H, Watanabe M, Shimizu K, Zou S, LaGraize SC, et al. Glial-cytokine-neuronal interactions underlying the mechanisms of persistent pain. $J$ Neurosci. 2007;27(22):6006-18. PMid:17537972. http://dx.doi.org/10.1523/

JNEUROSCI.0176-07.2007.

37. Melzack R, Wall PD. Pain mechanisms: a new theory. Science. 1965;150(3699):9719. PMid:5320816. http://dx.doi. org/10.1126/science.150.3699.971.

38. Baba H, Ji RR, Kohno T, Moore KA, Ataka T, Wakai A, et al. Removal of GABAergic inhibition facilitates polysynaptic: a fiber-mediated excitatory transmission to the superficial spinal dorsal horn. Mol Cell Neurosci. 2003;24(3):81830. PMid:14664828. http://dx.doi. org/10.1016/S1044-7431(03)00236-7.

39. Hwang JH, Yaksh TL. The effect of spinal GABA receptor agonists on tactile allodynia in a surgicallyinduced neuropathic pain model in the rat. Pain. 1997;70(1):1522. PMid:9106805. http://dx.doi. org/10.1016/S0304-3959(96)03249-6.

40. Malan TP JR, Mata HP, Porreca F. Spinal GABA(A) and GABA(B) receptor pharmacology in a rat model of neuropathic pain. Anesthesiology. 2002;96(5):1161-7. PMid:11981157. http://dx.doi.org/10.1097/00000542200205000-00020.

41. Stern P. Glee for glia. Science. 2010;330(6005):773. PMid:21051627. http://dx.doi.org/10.1126/ science.330.6005.773.

42. Colton C, Wilcock DM. Assessing activation states in microglia. CNS Neurol Disord Drug Targets. 2010;9(2):174-91. PMid:20205642. http://dx.doi.org/10.2174/1871527107 91012053.

43. Woolf CJ, Allchorne A, SafiehGarabedian B, Poole S. Cytokines, nerve growth factor and inflammatory hyperalgesia: the contribution of tumour necrosis factor alpha. $\mathrm{Br}$ J Pharmacol. 1997;121(3):41724. PMid:9179382. http://dx.doi. org/10.1038/sj.bjp.0701148.

44. LaMotte CC, Kapadia SE, Arsenault K, Wolfe M. Deafferentationinduced expression of GAP-43, NCAM, and NILE in the adult rat dorsal horn following pronase injection of the sciatic nerve. Somatosens Mot Res. 1995;12(1):719. PMid:7571944. http://dx.doi. org/10.3109/08990229509063143.

45. lida T, Liu S, Levitt RC, Candiotti KA, Lubarsky DA, Hao S. CPEB plays an important role in the HIV neuropathic pain state through spinal ROS and Wnt signaling. J Neuroimmune Pharmacol. 2015;10(2):S75-6.

46. Van der Watt JJ, Wilkinson KA, Wilkinson RJ, Heckmann JM. Plasma cytokine profiles in HIV-1 infected patients developing neuropathic symptoms shortly after commencing antiretroviral therapy: a case-control study. BMC Infect Dis. 2014;14(1):71. PMid:24512313. http://dx.doi. org/10.1186/1471-2334-14-71.

47. Zheng $X$, Ouyang H, Liu S, Mata M, Fink DJ, Hao S. TNFa is involved in neuropathic pain induced by nucleoside reverse transcriptase inhibitor in rats. Brain Behav Immun. 2011;25(8):1668-76. PMid:21741472. http://dx.doi.org/10.1016/j. bbi.2011.06.010

48. Zheng W, Huang W, Liu S, Levitt RC, Candiotti KA, Lubarsky DA, et al. IL-10 mediated by herpes simplex virus vector reduces neuropathic pain induced by HIV gp120 combined with ddC in rats. Mol Pain. 2014;10(1):49. PMid:25078297. http://dx.doi. org/10.1186/1744-8069-10-49.

49. Lu L, Dong H, Liu G, Yuan B, Li Y, Liu H. The Protective Effects of IGF-1 on Different Subpopulations of DRG Neurons with Neurotoxicity Induced by gp120 and Dideoxycytidine In Vitro. Biomol Ther (Seoul). 2014;22(6):5329. PMid:25489421. http://dx.doi. org/10.4062/biomolther.2014.036.

50. Keltner JR, Fennema-Notestine C, Vaida F, Wang D, Franklin DR, Dworkin RH, et al. HIV-associated distal neuropathic pain is associated with smaller total cerebral cortical gray matter. J Neurovirol. 2014;20(3):20918. PMid:24549970. http://dx.doi. org/10.1007/s13365-014-0236-8.

51. Kallianpur AR, Jia P, Ellis RJ, Zhao Z, Bloss C, Wen W, et al. Genetic variation in iron metabolism is associated with neuropathic pain and pain severity in HIV-infected patients on antiretroviral therapy. PLoS One. 2014;21(8):e103123. PMid:25144566. http://dx.doi.org/10.1371/journal. pone. 0103123 .

52. Ellis R, Letendre $S$, Rosario $D$, Gelman B, Clifford D, Simpson $D$, et al. A common polymorphism in the comt gene confers an increased risk of neuropathic pain in HIV sensory neuropathy. Neurology. 2012;78(Meeting Abstracts 1):1. http://dx.doi.org/10.1212/WNL.78.1_ MeetingAbstracts.\$37.006. 
53. Ellis RJ, Rosario D, Clifford D, McArthur JC, Simpson D, Alexander $\mathrm{T}$, et al. Risk factors for incident neuropathic pain in HIV infection: the Charter study. J Neurovirol. 2009;15:27.

54. Taylor BK. Pathophysiologic mechanisms of neuropathic pain. Curr Pain Headache Rep. 2001;5(2):15161. PMid:11252149. http://dx.doi. org/10.1007/s11916-001-0083-1.

55. Baron R. Mechanisms of disease: neuropathic pain: a clinical perspective. Nat Clin Pract Neurol. 2006;2(2):95-106. PMid:16932531. http://dx.doi.org/10.1038/ ncpneuro0113.

56. Urban MO, Gebhart GF. Supraspinal contributions to hyperalgesia. Proc Natl Acad Sci USA. 1999;96(14):768792. PMid:10393881. http://dx.doi. org/10.1073/pnas.96.14.7687.

57. Coderre TJ, Katz J. Peripheral and central hyperexcitability: differential signs and symptoms in persistent pain. Behav Brain Sci. 1997;20(3):404-19, discussion 435513. PMid:10097003. http://dx.doi. org/10.1017/S0140525X97251484.

58. Vranken JH. Mechanisms and treatment of neuropathic pain. Cent Nerv Syst Agents Med Chem. 2009;9(1):71-8. PMid:20021340. http://dx.doi.org/10.2174/1871524097 87601932.

59. National Institutes of Health. National Institute of Neurological Disorders and Stroke (NINDS). Peripheral neuropathy fact sheet. New York: 2012. [citado em 12 Mar 2015]. Disponível em: http://www. neuromodulation.com/fact_sheet painful_peripheral_neuropathy

60. Cornblath DR, McArthur JC. Predominantly sensory neuropathy in patients with AIDS and AIDS-related complex. Neurology. 1988;38(5):7946. PMid:2834669. http://dx.doi. org/10.1212/WNL.38.5.794.

61. Schifitto G, McDermott MP, McArthur JC, Marder K, Sacktor N, Epstein $\mathrm{L}$, et al. Incidence of and risk factors for HIV-associated distal sensory polyneuropathy. Neurology. 2002;58(12):1764-8. PMid:12084874. http://dx.doi.org/10.1212/ WNL.58.12.1764.

62. Ellis RJ, Rosario D, Clifford DB, McArthur JC, Simpson D, Alexander $\mathrm{T}$, et al. Continued high prevalence and adverse clinical impact of human immunodeficiency virus-associated sensory neuropathy in the era of combination antiretroviral therapy: the CHARTER Study. Arch Neurol. 2010;67(5):552-8. PMid:20457954. http://dx.doi.org/10.1001/ archneurol.2010.76.

63. Ebirim LN, Otokwala JG. Inadequate pain relief in ambulatory patients with human immunodeficiency virus disease in Port Harcourt. HIV AIDS (Auckl). 2013;5:199203. PMid:23976866. http://dx.doi. org/10.2147/HIV.S45150.

64. Malvar J, Vaida F, Sanders CF, Atkinson JH, Bohannon W, Keltner $\mathrm{J}$, et al. Predictors of new-onset distal neuropathic pain in HIV-infected individuals in the era of combination antiretroviral therapy. Pain. 2015;156(4):731-9. PMid:25659067. http://dx.doi.org/10.1097/01.j.pa in.0000461252.75089.bf.

65. Keltner J, Connolly C, Vaida F, Franklin D, FitzSimons C, Corkran $\mathrm{S}$, et al. HIV-associated distal neuropathic pain is associated with smaller ventral posterior cingulate cortex. J Pain. 2013;14:4:S30.

66. Sanna MD, Peroni D, Quattrone A, Ghelardini C, Galeotti N. Spinal RyR2 pathway regulated by the RNA-binding protein HuD induces pain hypersensitivity in antiretroviral neuropathy. Exp Neurol. 2015;267:5363. PMid:25765490. http://dx.doi. org/10.1016/j.expneurol.2015.02.036.

67. Husstedt IW, Oliver S, Reichelt D, Gehring E, Kreutzkamp E, Philipp C. Topic capsaicin for treatment of HIV-associated polyneuropathy. Reg Anesth Pain Med. 2013;38:1. PMid:23263207.

68. Nasirinezhad F, Jergova S, Pearson JP, Sagen J. Attenuation of persistent pain-related behavior by fatty acid amide hydrolase (FAAH) inhibitors in a rat model of HIV sensory neuropathy. Neuropharmacology. 2015;95:100-9. PMid:25486617. http://dx.doi.org/10.1016/j. neuropharm.2014.11.024.

69. Obiako OR, Abdu-Aguye I, Ogunniyi A. Effect of stavudine-based antiretroviral therapy on the severity of polyneuropathy in HIVIAIDS patients: a preliminary report from Zaria, Northern Nigeria. West Afr J Med. 2011;30(5):354-8. PMid:22752824.
70. Garrow AP, Xing M, Vere J, Verrall B, Wang L, Jude EB. Role of acupuncture in the management of diabetic painful neuropathy (DPN): a pilot RCT. Acupunct Med. 2014;32(3):242-9. PMid:24657491. http://dx.doi.org/10.1136/ acupmed-2013-010495.

71. Ngernyam N, Jensen MP, Arayawichanon $\mathrm{P}$, Auvichayapat $\mathrm{N}$, Tiamkao S, Janjarasjitt S, et al. The effects of transcranial direct current stimulation in patients with neuropathic pain from spinal cord injury. Clin Neurophysiol. 2015;126(2):38290. PMid:25027640. http://dx.doi. org/10.1016/j.clinph.2014.05.034.

72. Galhardoni R, Correia GS, Araujo H, Yeng LT, Fernandes DT, Kaziyama $\mathrm{HH}$, et al. Repetitive transcranial magnetic stimulation in chronic pain: a review of the literature. Arch Phys Med Rehabil. 2015;96(4 Suppl):S156-72. PMid:25437106. http://dx.doi. org/10.1016/j.apmr.2014.11.010.

73. Dobson JL, McMillan J, Li L. Benefits of exercise intervention in reducing neuropathic pain. Front Cell Neurosci. 2014;8:102. PMid:24772065. http:// dx.doi.org/10.3389/fncel.2014.00102.

74. Tumusiime DK, Stewart A, Venter FW. Effect of physiotherapeutic exercises on peripheral neuropathy, functional limitations of lower extremity and quality of life in people with HIV. Physiotherapy (United Kingdom). 2015;101:eS1547-8.

75. Knezevic NN, Candido KD, Rana S, Knezevic I. The use of spinal cord neuromodulation in the management of HIV-related polyneuropathy. Pain Physician. 2015;18(4):E643-50. PMid:26218955.

76. Chang Chien GC, Knezevic NN, Candido KD. Spinal cord neuromodulation in the management of HIV-related polyneuropathy: case report. PM R. 2014;6(9):S373-4. http:// dx.doi.org/10.1016/j.pmrj.2014.08.901.

77. Puppala VK, Nick Knezevic N, Candido K. Spinal cord stimulation for management of HIV-related polyneuropathy. Pain Med. 2011;12(522):3. PMid:21223491.

78. Heutink M, Post MW, Overdulve CW, Pfennings LE, van de Vis W, Vrijens $\mathrm{NL}$, et al. Which pain coping strategies and cognitions are associated with outcomes of a cognitive behavioral intervention for neuropathic pain after spinal cord injury? Top Spinal 
Cord Inj Rehabil. 2013;19(4):33040. PMid:24244098. http://dx.doi. org/10.1310/sci1904-330.
79. Silveira MP, Maurer P, Guttier MC, Moreira LB. Factors associated with therapeutic success in HIV-positive individuals in southern Brazil. $J$
Clin Pharm Ther. 2015;40(2):1925. PMid:25422132. http://dx.doi. org/10.1111/jcpt.12233.

Recebido: Jul 06, 2016 Aceito: Set 06, 2016 